\title{
ExOrthist: a tool to infer exon orthologies at any evolutionary distance
}

Yamile Márquez ${ }^{1 *}{ }^{*}$, Federica Mantica ${ }^{1 \dagger}$, Luca Cozzuto $^{1}$, Demian Burguera ${ }^{1,3}$, Antonio Hermoso-Pulido ${ }^{1}$, Julia Ponomarenko ${ }^{1,2}$, Scott W. Roy ${ }^{4}$ and Manuel Irimia ${ }^{1,2,5^{*}}$ (D)

* Correspondence: ymarquez1710@ gmail.com; mirimia@gmail.com †Yamile Márquez and Federica Mantica are co-first authors and contributed equally to this work. ${ }^{1}$ Centre for Genomic Regulation, Barcelona Institute of Science and Technology, Dr. Aiguader, 88, 08003 Barcelona, Spain

Full list of author information is available at the end of the article

\begin{abstract}
Several bioinformatic tools have been developed for genome-wide identification of orthologous and paralogous genes. However, no corresponding tool allows the detection of exon homology relationships. Here, we present ExOrthist, a fully reproducible Nextflow-based software enabling inference of exon homologs and orthogroups, visualization of evolution of exon-intron structures, and assessment of conservation of alternative splicing patterns. ExOrthist evaluates exon sequence conservation and considers the surrounding exon-intron context to derive genomewide multi-species exon homologies at any evolutionary distance. We demonstrate its use in different evolutionary scenarios: whole genome duplication in frogs and convergence of Nova-regulated splicing networks (https://github.com/biocorecrg/ ExOrthist).
\end{abstract}

Keywords: Alternative splicing, Orthology, Paralogy, Intron-exon structures

\section{Background}

One of the most fascinating innovations of eukaryotic organisms was the evolution of gene structures composed of exons-pre-mRNA sequences joined and translated into proteins-and interspersed introns-removed from the pre-mRNA through the process of splicing (Fig. 1A). Such innovation greatly increased the evolutionary potential of eukaryotic genomes. First of all, although rarely, changes in the exon-intron structure can modify the function of conserved genes (e.g., [1, 2]). More commonly, many eukaryotic genes acquire the ability to differentially combine their exons and introns into multiple isoforms through the process of alternative splicing. In particular, the inclusion or removal of an entire exon from an isoform (exon skipping) is the most common type of alternative splicing in metazoans and the main contributor to alternative splicing-driven proteome diversification [3]. Exon skipping has been shown to be fastevolving [4-6], although some cassette exons are exceptionally conserved at the genomic and regulatory level [7]. Furthermore, in several cases, novel alternative splicing events have been linked to organismal innovations [8, 9]. Thus, exon-intron structure and alternative splicing patterns are two relevant intragenic features that have the

(c) The Author(s). 2021 Open Access This article is licensed under a Creative Commons Attribution 4.0 International License, which permits use, sharing, adaptation, distribution and reproduction in any medium or format, as long as you give appropriate credit to the original author(s) and the source, provide a link to the Creative Commons licence, and indicate if changes were made. The images or other third party material in this article are included in the article's Creative Commons licence, unless indicated otherwise in a credit line to the material. If material is not included in the article's Creative Commons licence and your intended use is not permitted by statutory regulation or exceeds the permitted use, you will need to obtain permission directly from the copyright holder. To view a copy of this licence, visit http://creativecommons.org/licenses/by/4.0/. The Creative Commons Public Domain Dedication waiver (http://creativecommons.org/publicdomain/zero/1.0/) applies to the data made available in this article, unless otherwise stated in a credit line to the data. 


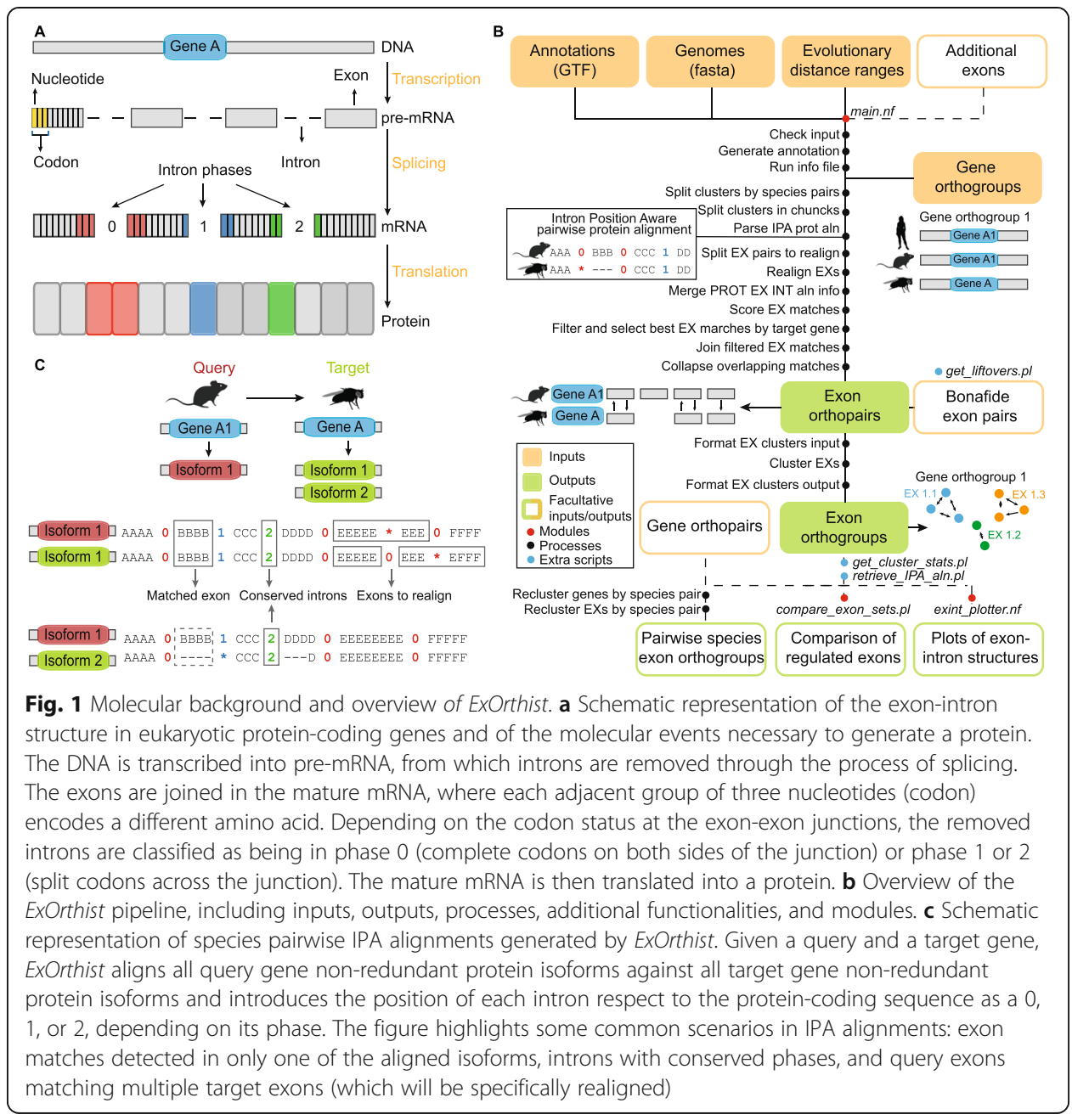

potential to mediate functional diversification within conserved genes. However, how to link such features to function remains an open challenge [10, 11].

Comparative genomics has been an important tool to link whole genes to functions by inferring gene presence/absence in different species through the identification of evolutionarily related gene orthologs (originated by a speciation event) and paralogs (derived from a duplication event). While orthologs usually maintain the ancestral function, paralogs are free to mutate and evolve new functions, as long as the ancestral role is preserved $[12,13]$. Remarkably, although multiple bioinformatic tools have been developed to identify homology relationships at the gene level [14-18], and some are emerging to investigate the conservation of transcript isoforms and/or splicing patterns [19-21], no corresponding tool currently exists to infer homology relationships at the exon level, particularly over deep evolutionary timescales. Nonetheless, even in the absence of general-purpose tools, a variety of studies have underscored the importance of this approach. First, in much the same manner as origins of novel genes have been linked to evolution of new molecular functions, de novo origins of alternatively spliced exons have been associated with evolution of novel gene functions [7, 8]. Second, in the same way as gene paralogs frequently evolve new functions, duplicated paralogous exons have been shown to provide novel functional properties to the host gene [22, 
23]. Moreover, even in cases in which new functions do not emerge, mutually exclusive splicing of duplicated exon pairs can lead to the specialization of alternative transcripts for distinct pre-existing functions, mirroring the impact of whole gene duplication [24].

General tools for assigning and studying orthologous and paralogous exons are thus crucial to properly investigate the evolution of gene structures and alternative splicing. For a few model organisms, it is possible to confidently infer pairs of orthologs at short evolutionary distances (e.g., within mammals or within fruitflies) using liftOver [25]. Besides the restriction to short evolutionary distances, this approach relies on the availability of precomputed UCSC pairwise chain alignments and can be applied only to one species pair at a time (i.e., multi-species orthogroups cannot be inferred). Moreover, liftOver derives exon orthopairs exclusively based on interpolation of the target exon sequence within whole genome alignments, which has two main limitations. First, it does not take into account the preservation of the two neighboring intron positions, whose ancestrality is a requisite for "full" homology of a pair of exons. Second, it does not assess the conservation of the upstream and downstream exons, which helps to further support homology of the neighboring intron positions.

To overcome these limitations and fulfill the major need for a software for exon homology inference, we have developed ExOrthist, which uses Intron Position Aware (IPA) protein alignments to infer species pairwise exon homologs and multi-species orthogroups, with substantial flexibility to allow fine tuning according to evolutionary distance. ExOrthist has three modules. The main module is a Nextflow-based pipeline [26] that infers exon homologies using an innovative approach, namely evaluating three features: conservation of the neighboring intron positions (with respect to the proteincoding sequence), sequence and length conservation of the query exons, and sequence conservation of the upstream/downstream exons. Second, the exint_plotter module is also a Nextflow-based pipeline that allows the visualization of evolutionary conservation and of changes in exon-intron structure among gene homologs of interest. Third, the compare_exon_sets module assesses the conservation of alternative splicing patterns between pairs of species. Here, we provide the first description of ExOrthist. We first give an overview of the pipeline (schematized in Fig. 1B), describe how we selected the default conservation cut-offs for three evolutionary distance ranges, and compare ExOrthist and liftOver performances on a set of closely related mammalian species. Then, we illustrate the use of ExOrthist to (i) reconstruct paralogous relationships and asymmetric evolution after a whole genome duplication event in the Xenopus lineage; and (ii) compare Nova-regulated exons in mouse and fruitfly, unveiling a substantial degree of convergent evolution of Nova regulation, which has independently targeted orthologous genes in both lineages. All ExOrthist modules are freely available at https://github. com/biocorecrg/ExOrthist.

\section{Results}

Overview of ExOrthist main module

The ExOrthist main module infers exon homologies within a set of species for which annotation files (in GTF format) and genome files (in fasta format) are provided. Moreover, it is possible to provide additional non-annotated exons identified from RNA-seq data by any splicing quantifier (e.g., [27-31]). ExOrthist derives exon homologies at two 
different levels: (i) exon orthopairs, representing pairs of homologous exons from two different species, and (ii) exon orthogroups, which include all the exons sharing a common ancestor (and thus both orthologs and paralogs) for multiple species. As such, orthopairs and orthogroups provide complementary information that is necessary for the complete reconstruction of exon evolutionary relationships. Since, by definition, exon orthopairs/orthogroups are derived from evolutionarily related genes, ExOrthist also requires as input a set of gene orthogroups for the species of interest (e.g., generated by Orthofinder [17], Broccoli [18], or similar tools). While ExOrthist is designed to infer genome-wide exon homologies, it can also be restricted to one/few genes of interest by providing only a subset of gene orthogroups as input. ExOrthist utilizes a set of conservation cut-offs (number of conserved intron positions, minimum exon sequence similarity, minimum exon length ratio, minimum global protein similarity; see below), which can be differentially fine-tuned for three evolutionary distance ranges (short, medium, and long). Thus, the evolutionary distance range between each pair of considered species also has to be specified (see online README for further details on the input).

Once launched, ExOrthist extracts exon/intron-related information from the annotation files of all the species considered, and then it performs the subsequent steps for each pair of species. First, it generates IPA alignments for all non-redundant protein isoforms belonging to the same gene orthogroup for each species pair (species1-species2) (Fig. 1C). This all-vs-all isoform comparison is necessary because a selection of representative isoforms might leave some exon orthologs undetected simply because they are not included in any of the selected protein isoforms. Considering species1 as query and species2 as target (and vice versa), ExOrthist then parses all pairwise isoform alignments. For each query exon, it first identifies the best matching exon in each target isoform based on the global protein alignment and sequence similarity. Next, for each best exon match per target gene isoform, ExOrthist evaluates its potential homology with the query exon based on the conservation cut-offs provided (see below). If the comparison between the query and target exon passes all the cut-offs, they are considered as putative homologs. Then, to select the best exon match for each target gene among all putative homologs (from the different isoforms), a global score [0-1] is calculated as the sum of five partial scores reflecting the conservation of the different features of the exon-intron context that were separately evaluated to assess homology: (1, 2) conservation of the immediately upstream and downstream intron positions and phases, (3) conservation of the query exon sequence and $(4,5)$ conservation of the immediately upstream and downstream exon sequences. The exon match (i.e., query exon - target isoform) with the highest global score among those passing all conservation cut-offs is selected as best match for that target gene, and the matching query and target exons are thereafter considered an orthopair. Importantly, while the ExOrthist logic requires a query exon to match a unique exon in the target gene, each exon in the target gene can potentially be matched by multiple query exons: this setting captures cases of in-tandem exon duplication within the same gene while preserving the information about which duplicated exon is more similar to the pro-ortholog in the other species. Furthermore, the user has the possibility to provide custom bona fide exon orthopairs, which will be directly incorporated into the generation of the exon orthogroups. This can be obtained from manual curation (e.g., through literature searches), or in a 
genome-wide manner using the liftOver tool. For this purpose, ExOrthist provides an additional script to perform liftOver between any pair of species for which UCSC liftOver files are available.

After deriving species pairwise exon homology relationships, ExOrthist infers multispecies exon orthogroups. The exon orthopairs for all the species pairs are joined and translated into a directed graph using the $\mathrm{R}$ igraph package [32], where an edgebetweenness algorithm selects the optimal topology, i.e., with high intra-community but low inter-community connections. The exon communities identified in the optimal topology correspond to the exon orthogroups returned by ExOrthist. Finally, ExOrthist offers the additional functionality to generate orthogroups restricted to two species when species pairwise gene orthopairs are provided as extra input.

\section{ExOrthist calibration and benchmarking}

ExOrthist allows the user to differentially specify a set of conservation cut-offs across three evolutionary distance ranges (short, medium, long): (a) minimum global protein similarity ("prot_sim"); (b) minimum number of conserved intron positions $(0,1$ or 2; "int_num"); (c) minimum exon sequence similarity ("ex_seq"); and (d) minimum exon length ratio (shortest_exon/longest_exon; "ex_len"). This flexibility in the definition of cut-offs is particularly relevant, since the use of fixed conservation parameters across different evolutionary distances would result in noisy homology calls for closely related species and incomplete homolog detections for more distant species.

Minimum global protein similarity, or cut-off (a), is the minimum sequence similarity over the entire pairwise protein alignment for a pair of protein isoforms to be considered for further comparisons. While it has no effect on the downstream analyses, it avoids processing spurious gene orthologs or poorly annotated isoforms. The minimum number of conserved introns, or cut-off (b), refers to the requirement for both, one or none of the neighboring intron positions to be conserved, and hence inferred to be ancestral (Fig. 1A). A pair of introns from two genes is considered to be in conserved positions if they have the same phase (i.e., interrupt a codon in the same nucleotide position; Fig. 1A) and are in a comparable location of the protein alignment. In particular, the maximum distance allowed between the two conserved intron positions in the protein alignment depends on the local protein sequence similarity and percentage of unaligned residues (see "Methods") [33]. Other features, such as intronic length or intronic sequence similarity, are not taken into consideration to assess conservation of intron positions. In this study, we consider homologous internal exons only those for which both neighboring intron positions are conserved; therefore, this cut-off is always set to 2 in all the following analyses. However, by lowering the number of required conserved introns to one, internal exons sharing a single intron position can also be accommodated in the final orthologies.

ExOrthist also requires a minimum exon protein sequence similarity, or cut-off (c), for both the query exon as well as the neighboring exons, and a minimum exon length ratio, or cut-off $(\mathrm{d})$, between the query exon and its match (shorter/longer). Since these two cut-offs are the main determinants of exon homology calls, we aimed at defining the most optimal combination of cut-offs for each evolutionary distance range. For this purpose, we chose human and mouse, human and zebrafish, and human and drosophila as representative species pairs for short, medium, and long evolutionary distances, 


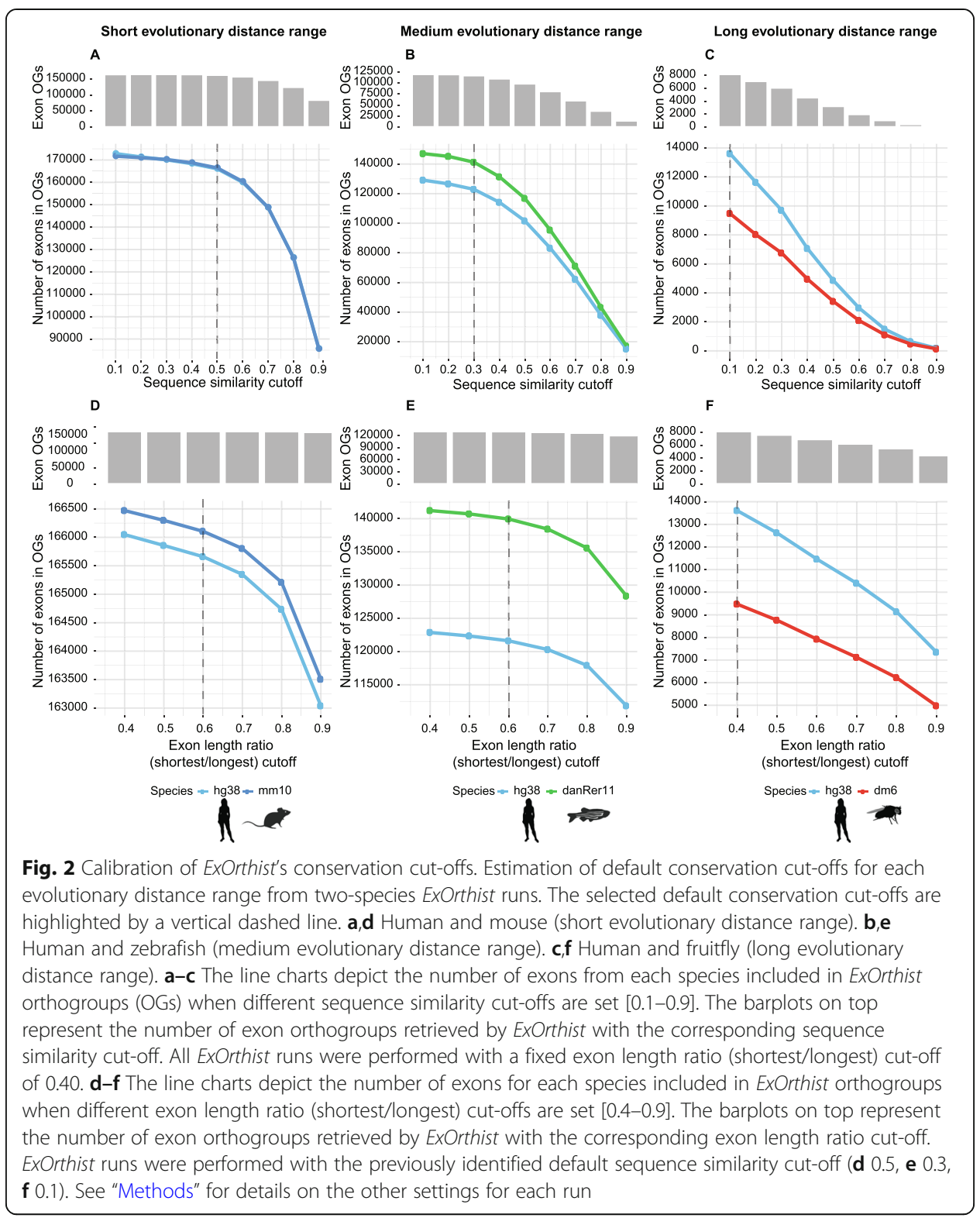

respectively. We first evaluated ExOrthist's performance at a fixed exon length ratio (0.40) and with a minimum exon protein sequence similarity ranging from 0.1 to 0.9 (Fig. 2A-C). Here, it is expected that lower cut-offs will retrieve higher numbers of inferred homologous relationships. Therefore, we specifically aimed at identifying the highest sequence similarity cut-off yielding nearly maximal detection sensitivity. This means the cut-off at which the vast majority of potential exon homologs would be included in the final orthogroups but for which lower cut-offs would not substantially increase the number of detected conserved exons. In the case of short and medium evolutionary distance ranges, the number of conserved exons included in orthogroups reached saturation at sequence similarity 0.5 and 0.3 , respectively, with lower cut-offs leading to only minor increases in the number of exons included in the orthogroups. Thus, we selected 0.5 and 0.3 as the default minimum sequence similarity cut-offs for 
short and medium evolutionary distance ranges. For the human-drosophila comparison (long evolutionary distance), it was not possible to identify a saturation point, suggesting that a substantial fraction of exonic sequences has evolved beyond recognition. We therefore set the default value for minimum sequence similarity for long evolutionary distance ranges to 0.1 .

Next, using these default cut-offs for the minimum sequence similarity, we evaluated the effect of varying the minimum exon length ratio from 0.4 to 0.9 for each evolutionary distance range. The goal of this analysis is thus to identify the optimal maximum relative length difference allowed between the two homologous exons for each evolutionary distance range. As above, the number of inferred exon homologs and orthogroups saturated at an intermediate value for short and medium, but not for long, evolutionary distance ranges (Fig. 2D-F). Therefore, we set the default values for minimum exon length ratio to 0.6 for short and medium and 0.4 for long evolutionary distance ranges, respectively. Data on the computing performance of ExOrthist for each type of run (short, medium, long), as well as for combinations of three or four species, are provided in Additional file 1: Figure S1 and in the respective Nextflow reports [34]. As an example, an analysis involving two vertebrate species with many annotated isoforms (e.g., human and zebrafish, with $\sim 93 \mathrm{~K}$ and $\sim 46 \mathrm{~K}$ protein-coding transcripts, respectively), takes $\sim 1 \mathrm{~h}$ of run time in a CPU cluster, requiring $\sim 99 \mathrm{~h}$ of CPU time, $4.5 \mathrm{~GB}$ of RAM memory, and less than $10 \mathrm{~GB}$ of disk space. Providing precomputed IPA alignments from previous ExOrthist runs (using the option --prevaln) reduces the necessary CPU time to less than $25 \mathrm{~h}$, saving more than $75 \%$ of the required computation time. We also implemented a configuration file for running the whole analysis on the Amazon cloud. For this test case, we uploaded input data into a S3 Bucket and used an AWS Batch spot queue with a bid percentage of 50\% using optimal instance type nodes. The test run took $\sim 3.5 \mathrm{~h}$ to complete, with $\sim 31 \mathrm{CPU}$ hours and a total cost below 3 EUR. Thus, there is no need for having a powerful HPC for running an analysis with well-annotated vertebrate species with ExOrthist.

We also aimed at assessing the sensitivity and accuracy of ExOrthist's homology calls. Although there is currently no available software to infer equivalent exon homology relationships, we compared the output of ExOrthist with that obtained using a liftOverbased approach ([35] and see "Methods"). We generated exon orthogroups for all annotated exons in human, mouse, and cow using ExOrthist with default cut-offs for short evolutionary distance. ExOrthist retrieved 148,255 1:1:1 exon triplets (83.3\% of all exon orthogroups) and $25,832(14.5 \%)$ orthogroups with a missing species (40.1\% of which corresponded to cow) (Fig. 3A). Internal coding human exons showed higher levels of genomic conservation (i.e., had at least an ortholog in another species) than first and last exons (Fig. 3B), and, as expected, these conservation levels were strongly dependent on the average inclusion of the exon, with $>99 \%$ and $<18 \%$ of constitutive and cryptic internal exons having an ortholog in the other species, respectively (Fig. 3B). Importantly, the comparison with the liftOver-based pipeline showed a high degree of overlap (>97\%) among the exons recovered in the orthogroups by each approach (Fig. 3C), with discrepancies largely corresponding to differences in annotations of exon variants between species. Moreover, the concordance between the orthology calls was $\sim 100 \%$ (i.e., the same pairs of exons from species1 and species 2 were considered orthopairs by both methods) (Fig. 3C). Finally, we selected a simple gene orthogroup (MIS18A) to exemplify the output of the exint_plotter module (Fig. 3D). 


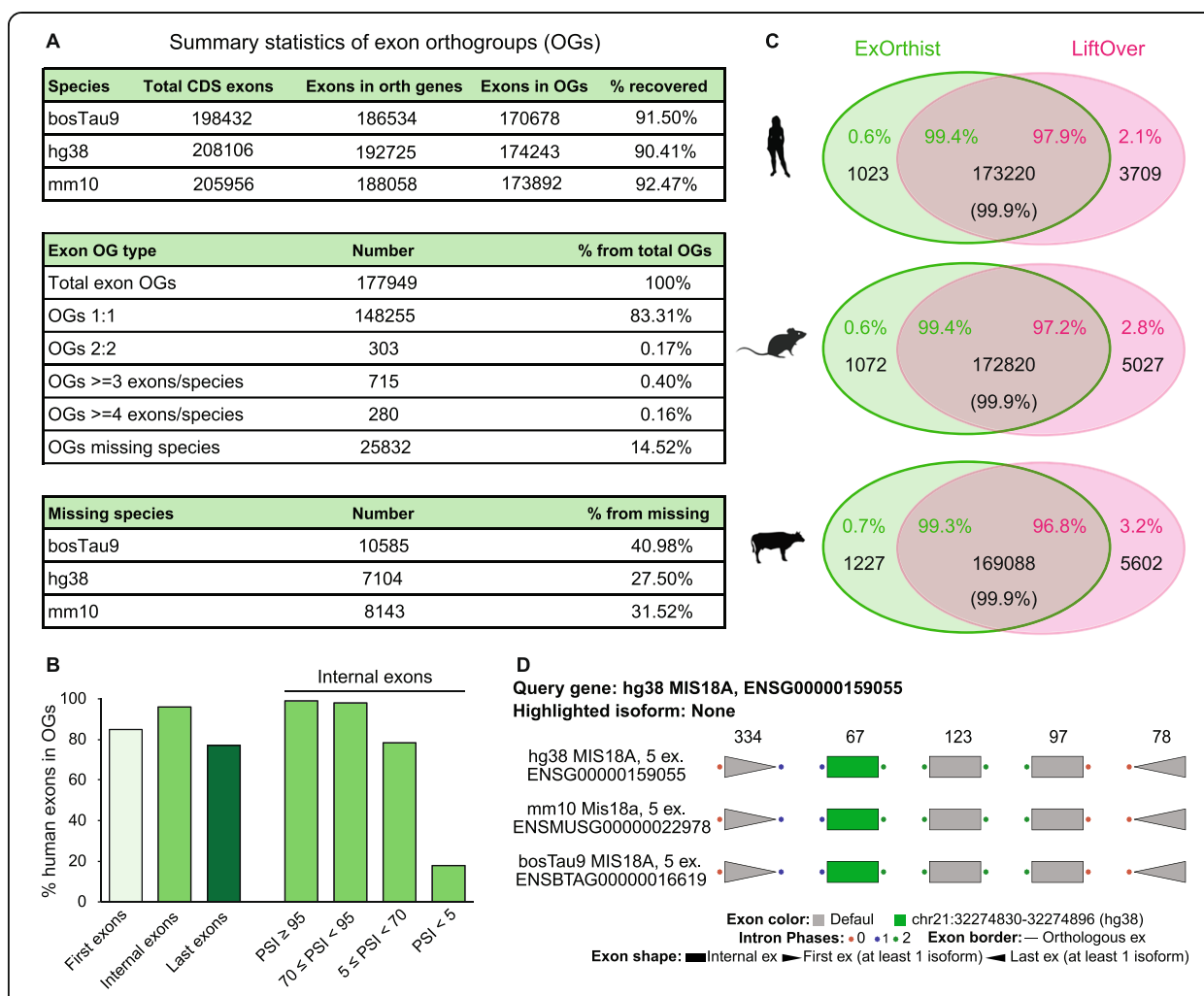

Fig. 3 ExOrthist output and benchmarking. a Statistics of the exon orthogroups (OG) from an ExOrthist main run on three mammalian species (human, mouse, cow) as generated by get_cluster_stats.pl. b Percentages of conserved human coding exons based on their location within the gene (first, internal, last) or on their average inclusion level (for internal exons only), as obtained from VastDB [29]. c Venn diagrams showing the number of exons (black) included in either ExOrthist orthogroups (green area), liftOver-based orthogroups (pink area) or both (intersection) for human, mouse, and cow. The percentages of shared and not-shared exons between ExOrthist and liftOver-based orthogroups are reported in the corresponding area. The percentage in brackets refers to the concordance between the 1:1 exon pairs in the target species (e.g., mouse and cow for human exons) retrieved by both methods. $\mathbf{d}$ Plot of the exon-intron structure of human MIS18A and its mouse and cow orthologs as generated by the exint_plotter module. Exons in the different species falling in the same exon clusters are vertically aligned, and a randomly chosen exon of interest is highlighted in green. Intron positions are depicted as colored dots, depending on the phase (0: red, 1: blue, 2: green). First and last exons are shown as arrows, and the length of the CDS portion of each human MIS18A exon (in nucleotides) is reported on top

\section{Using ExOrthist to investigate asymmetric exon evolution after gene duplication}

One important feature of ExOrthist is that it affords exon homology inferences not only for cases of clear 1:1 orthologs but also in scenarios with different levels of paralogy. Therefore, to assess this functionality, we performed an evolutionary exon comparison between Xenopus tropicalis and the allotetraploid Xenopus laevis. Since the whole genome duplication of $X$. laevis is relatively recent (17-18 million years ago), most of its genes can be readily assigned to one of the two ancestral subgenomes (named $\mathrm{L}$ and $\mathrm{S}$, respectively; Fig. 4A) [36]. Although both ancestral copies (homeologs) have been retained for multiple genes, a substantial fraction has asymmetrically retained a single copy, and this has most often corresponded to the one in the L subgenome (Fig. 4B) [36]. To investigate exon evolution after whole genome duplication, we used ExOrthist to generate exon orthogroups within a pre-defined set of 8806 X. leavis homeolog pairs and their respective $X$. tropicalis single orthologs (1:2 gene orthologs) [36]. ExOrthist identified 69,035 (88.0\%) 1:2 exon orthologs as well as 9377 (12.0\%) 1:1 relationships, 


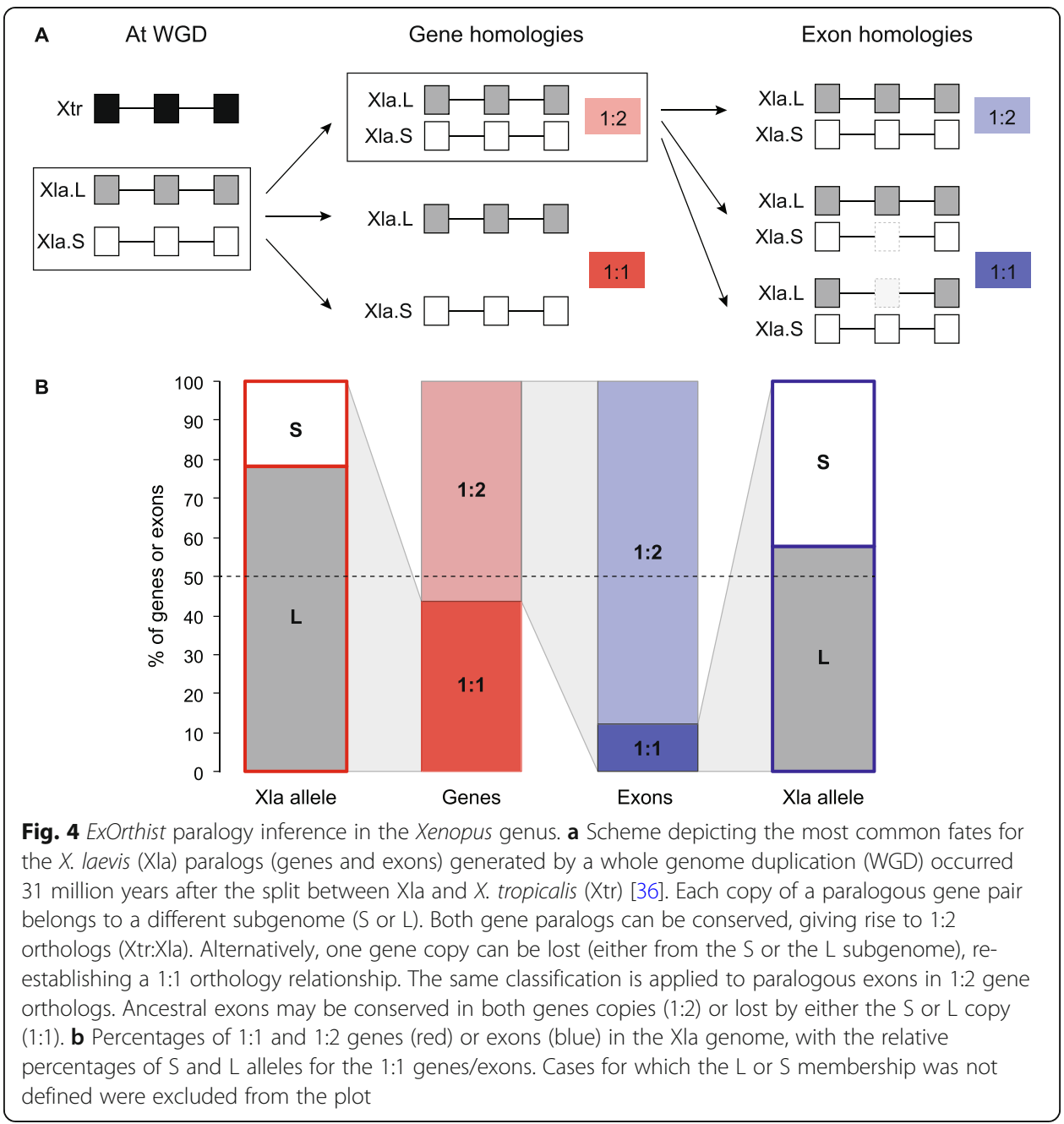

which indicate lack of detected exon homology for one gene homeolog in X. laevis after genome duplication (Fig. 4B). Interestingly, similar to the pattern of retention of gene duplicates, we found that gene copies from the L subgenome more often retained detected ancestral exon homology compared to the $\mathrm{S}$ subgenome (Fig. 4B; $57.7 \%, P=7.8 \mathrm{e}$ -48, two-sided Binomial test).

\section{Evaluation of genomic and regulatory conservation of alternatively spliced exon sets}

A major application of ExOrthist is the assessment of the evolutionary conservation of alternatively spliced exon networks (also referred to as exon programs). These networks consist of sets of exons that are regulated in a coordinated manner in a specific tissue (e.g., brain, muscle), usually through the action of tissue-enriched alternative splicing factors, and are known to play crucial roles in cellular differentiation and physiology $[37,38]$. Moreover, some sets of exons have been shown to be jointly misregulated in human pathologies, including cancer or mental disorders [39, 40], and the evolutionary comparison between human and model organisms is a powerful approach to identify potential pathogenic targets [41]. The ExOrthist compare_exon_sets module allows the evaluation of the evolutionary conservation of exon sets between two species from a 
genomic and regulatory perspective, both at the gene and exon level (Fig. 5A). At the gene level, ExOrthist assesses the proportion of exons in genes that have orthologs in the target species and the proportion of those orthologs that also harbor regulated exons. Similarly, exons are individually evaluated to infer what proportion have orthologs in the other species (referred to as "Genome conservation" [42]) and for what proportion those orthologs are also regulated ("Regulation conservation" [42]). Additionally, for all gene orthologs containing regulated exons in both species, the compare_exon_sets module performs a pairwise comparison to assess whether the exon pairs are as follows: (i) orthologs, (ii) best-hits (i.e., they are considered the best matching exon by the main module, but do not meet all the requirements to be considered proper homologs), (iii) non-orthologs, or (iv) unclear. In particular, ExOrthist relies on several aspects to confidently assign two exons as non-orthologs (Additional file 1: Figure S2), allowing the inference of evolutionarily independent alternative splicing patterns.

We used ExOrthist to compare sets of exons regulated by the splicing factor Nova in mouse and fruitfly. For this purpose, we ran vast-tools [29] to obtain changes in inclusion levels ( $\triangle$ PSIs) for all exons upon Nova2 depletion in mouse embryonic cortex [43]

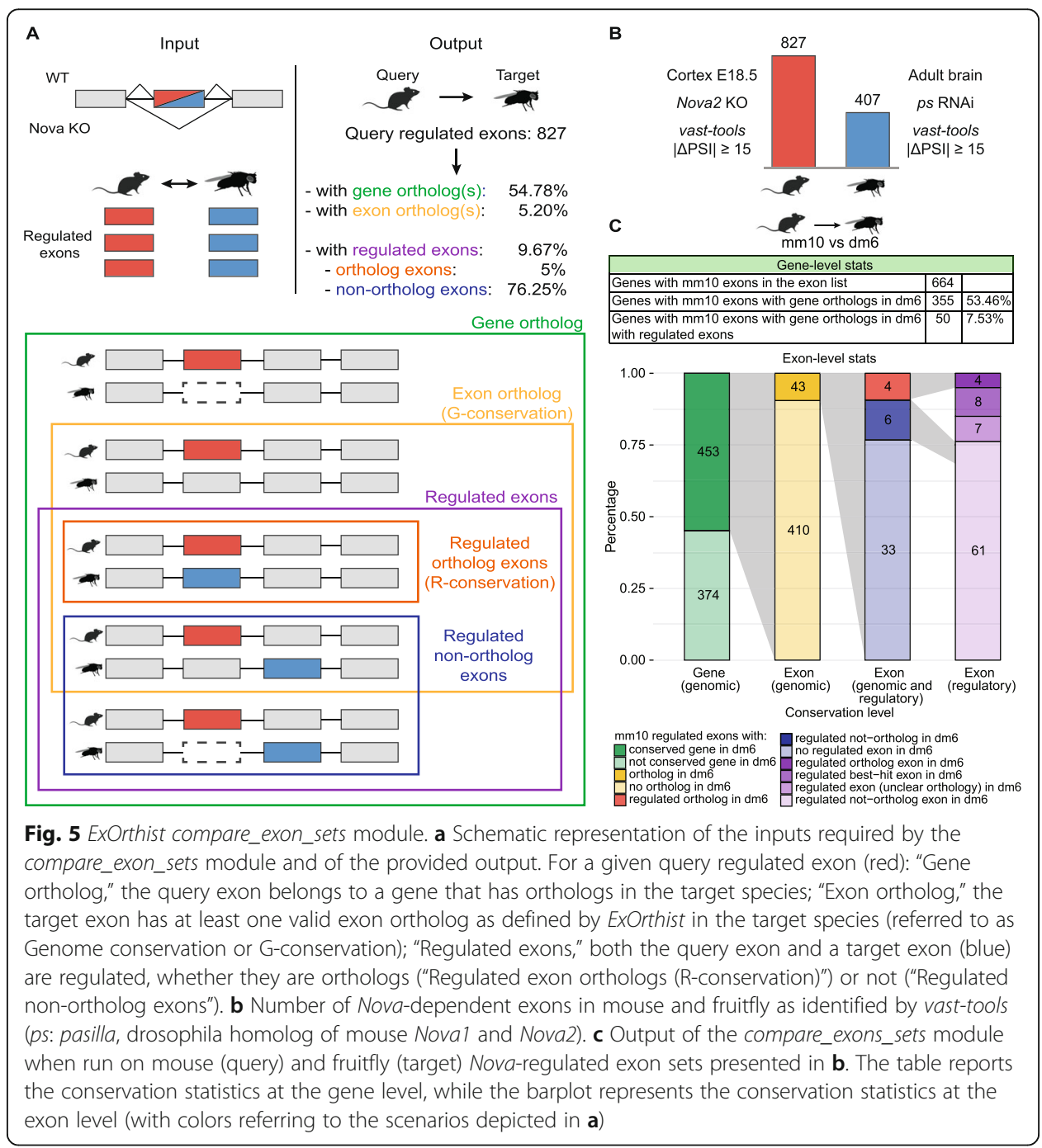


and upon knockdown of the fruifly ortholog (ps) in whole adult brains [44]; we then defined as Nova-dependent exons in each species those with a $|\Delta \mathrm{PSI}|>15$ (827 in mouse and 407 in fruifly, respectively; Fig. 5B). In parallel, we ran ExOrthist main for mouse and fruitfly with default cut-offs for the long evolutionary distance range and providing all exons evaluated in vast-tools as additional exons for each species through the --extraexons option. We then utilized the compare exon_sets module to compare the mouse and fruitfly Nova-dependent exon sets and identify conserved exons. Despite the expected low conservation [45, 46], ExOrthist identified 43 (5.2\%) mouse Nova-dependent exons sharing orthogroups with fruitfly exons, 4 of which were also impacted by Nova depletion in the insect species (Additional file 2: Table S1). However, the most remarkable finding of this investigation was evidence for shared Nova regulation of orthologous genes through regulation of non-orthologous exons: 80 mouse exons (in 50 genes) were in gene orthologs harboring Nova-dependent exons in fruitfly (77 exons in 41 genes), the vast majority of which (61/80 mouse exons, $76.3 \%)$ corresponded to non-orthologous exon pairs in the two species, compared to the 4 (5\%) cases with regulatory conservation and $8(10 \%)$ additional pairs that were best exon hits although they did not pass all the homology filters (Fig. 5C and Additional file 3: Table S2). The overlap of homologous genes was much higher than expected by chance $(P=1.7 \mathrm{e}-13$, one-sided Hypergeometric test). These results highlight a major evolutionary convergence of Nova-regulated exon networks impacting orthologous genes, as seen also for other splicing factor networks [46-48].

To gain further insights into the exon programs regulated by Nova in both lineages, we next performed an in-depth comparative analysis. First, we found that exons that were up- and down-regulated upon Nova disruption showed a similar neural depletion and enrichment, respectively, in both mouse and fruitfly (Additional file 1: Figure S3A,B). While Nova-regulated exons are well known to be neurally regulated in mammals [49], this had not been investigated in fruitflies, where ps expression is broader during embryo development [46, 50]. Second, we used matt [51] to evaluate over 50 splicing-related features in both species. Compared to non-regulated alternative exons and other exon sets in their corresponding species, Nova-regulated exons had overall similar features, including shorter exon lengths (Additional file 1: Figure S3C-G). A notable exception was the length of poly-pyrimidine tract of the upstream intron, which was longer in mouse but shorter than for other exon sets in fruitflies (Additional file 1: Figure S3H). Finally, we also used matt to identify enriched motifs from RNAbinding proteins potentially associated with Nova regulation. As expected, Nova-regulated exons from both species exhibited strong enrichment for canonical YCAY clusters in the expected positions based on the direction of regulation (Additional file 1: Figure S4A,B) [45, 52]. Interestingly, we also found enrichment for Rbfox-binding motifs downstream of mouse Nova-dependent exons (Additional file 1: Figure S4C), in line with previous reports [53]. However, this enrichment was not observed for flies, suggesting the co-regulation by both factors is lineage-specific (Additional file 1: Figure S4D). Conversely, Nova-regulated exons in Drosophila, but not in mouse, exhibited enrichment for motifs for IGF2BP2/Imp (Additional file 1: Figure S4E,F), an RNA-binding protein upregulated during early neuronal differentiation in both lineages (Additional file 1: Figure $\mathrm{S} 4 \mathrm{G}, \mathrm{H})$. 


\section{Discussion}

Exon gains and losses have the potential to drive diversification of gene structure and function across eukaryotes. Moreover, alternative splicing of specific exons can greatly expand transcriptome and proteome diversity, especially in metazoans. In this context, evolutionary conservation both at the genomic and the regulatory level of alternative splicing has been widely regarded as a proxy for functional relevance [42]. A key step to assess such conservation is the inference of exon homology relationships among species. However, while multiple tools have been developed for gene homology inference, there is currently no available software to derive exon orthology and paralogy relationships. Here, we have introduced ExOrthist, the first software specifically designed to infer exon homology relationships taking into account the unique characteristics of exon and intron evolution across different evolutionary distances. Therefore, unlike gene-oriented software, ExOrthist does not rely on comparisons of exon sequence similarity in isolation, but also makes use of the full intragenic context. For this reason, ExOrthist identifies potential exon homologs through global protein alignments and explicitly requires conservation of the neighboring intron positions as well as of individual exon sequences within these global alignments.

Evolution of intron positions has been extensively studied, revealing a generally low rate of intron gain and loss within most eukaryotic groups [54-57], despite some remarkable exceptions (e.g., $[58,59])$. Comparison among lineages showed conservation of relatively high fractions of ancestral introns in various clades, as well as substantial remodeling of intron-exon architectures [56,60]. Since, by definition, intron insertions define (and redefine) exons, conservation of ancestral intron positions is a requirement for true exon homology of any given exon pair. Thus, because of their very low rates of intron gains/losses, it is expected that a large fraction of exons is conserved within vertebrates (Fig. 3, [5, 61, 62]), but not between species with largely divergent intron-exon architectures (e.g., between mammals and dipterans; Fig. 5). That is, even if the compared coding sequences are orthologous, the actual exon entities encoding them may not be. To our knowledge, ExOrthist is the first framework that incorporates the conservation of intron positions and other features of the gene context to explicitly draw the fundamental distinction between conserved exon sequences and conserved exon entities. In order to clearly distinguish between these two scenarios, ExOrthist also provides the best hit for each exon within each target gene homolog, even when the target exon does not meet the necessary requirements to be considered a homolog. As we have shown in the case of Nova-regulated exons, this allows the identification of cases of convergent evolution of (regulated) alternative splicing, in which two exons that are not orthologous, even if sometimes they partially share orthologous sequences, are independently recruited to corresponding exon networks in two lineages.

Since ExOrthist allows users to work at any evolutionary distance and with species for which only a genome sequence and a standard gene annotation is available, our software can be used for both model and non-model organisms. For this reason, we envision that ExOrthist will enable unprecedented evolutionary studies, both in terms of species representation and biological questions, opening multiple new venues of research on genome evolution and alternative splicing. To name a few, these could include the identification of deeply conserved alternative splicing events, the study of the origin and evolution of tissue-specific exon networks, the investigation of intragenic evolutionary patterns, the inference of phylogenies based on bona fide exon orthologs, 
and the construction of large multi-species databases interconnected not only through gene homology relationships (e.g., Ensembl), but also through exon homology relationships (e.g., VastDB [29]).

\section{Conclusions}

ExOrthist is the first tool specifically designed to infer exon orthogroups and orthopairs across any evolutionary distance. It also contains additional modules to visualize conservation of intron-exon architectures and to assess evolutionary conservation of alternative splicing patterns. ExOrthist will thus facilitate research on genome evolution at the intragenic level as well as the identification of highly conserved, and thus more likely functional, alternatively spliced exons.

\section{Methods}

\section{Algorithm of ExOrthist main module}

The ExOrthist main module requires a minimum set of inputs (annotation files, genome files, evolutionary distance information, and gene orthogroups) to return the complete set of exon orthopairs and orthogroups for a chosen group of species. The following sections (A, B, C, D) describe the algorithm applied by ExOrthist, as well as the extra steps implemented when additional inputs are provided. The module relies on software and libraries stored within a docker image whose recipe is bundled together with the rest of the pipeline. The image is available at docker hub (https://hub. docker.com/r/biocorecrg/exon_intron_pipe) and is automatically retrieved by ExOrthist and eventually converted into a singularity image if the option -with-singularity is specified. To run the program, a user only needs to install Nextflow and one Linux container, either Docker or Singularity. Further details can be obtained in the README section at https://github.com/biocorecrg/ExOrthist.

\section{A. Input generation}

After checking for the presence of all required inputs and the consistency between gene orthogroups and the annotated gene IDs in the GTF files, ExOrthist generates files with annotation information for each considered species, which will be the input of all species pairwise comparisons (see online README for a detailed list of all the files). Although, by default, only annotated exons are considered, ExOrthist can integrate nonannotated exons into its homology inferences by providing a list of gene identifiers plus exon coordinates (e.g., exons identified by vast-tools [29] or any other software to detect and/or quantify alternative splicing). If non-annotated exons are provided, ExOrthist generates a new GTF file where a novel transcript for each of the non-annotated exons is introduced. ExOrthist first maps the upstream and downstream exons (provided in the input file) to the annotated transcripts. When both neighboring exons match the same coding transcript, this becomes the template of the novel transcript to which the non-annotated exon is added; otherwise, ExOrthist uses partial matches of the upstream or downstream exon to identify the template transcript and create the new annotation. 
ExOrthist allows the user to specify different conservation cut-offs depending on the evolutionary distances between the compared species, which have to be declared in a specific input file. In particular, four evolutionary cut-offs can be fine-tuned for three evolutionary distance ranges (short, medium, long): (a) minimum global protein sequence similarity for a pairwise isoform alignment to be considered for the orthology call, (b) minimum number of conserved intron positions, (c) minimum sequence similarity required for orthologous exons, and (d) minimum ratio between the lengths of an exon pair (length of the shortest/longest exon). Cut-offs (b), (c), and (d) will be utilized in the later steps of the pipeline to filter out non-homologous matches from the pool of candidate exon orthopairs for each pair of species (see section C).

\section{B. Parsing of IPA alignments}

ExOrthist then works by species pairs and within gene orthogroups. For each species pair and gene orthogroup, it first generates pairwise Intron Position Aware (IPA) alignments between all non-redundant protein isoforms of species1 vs. all non-redundant protein isoforms of species2 (Fig. 1C). Protein sequence alignments are generated using MAFFT v7 [63] and intron positions are introduced subsequently. Considering species1 as query and species2 as target (and vice versa), ExOrthist parses the IPA alignments to derive:

- Protein conservation information: species-wise percentages of sequence identity, sequence similarity (based on BLOSUM62), and gaps between the two proteins are calculated. Alignments are not considered for further processing unless the global sequence similarity (cut-off (a)) reaches the minimum protein similarity specified for the relative evolutionary distance (see section A) for both species, or twice the minimum protein similarity cut-off $(2 \times$ cut-off (a)) for one of the species.

- Exon conservation information: for each query exon, ExOrthist selects all exon matches in all target isoforms. In case of a query exon matching multiple target exons in the same isoform each covering $\geq 15 \%$ of the query exon sequence, each exon pair is specifically realigned and the best match is selected based on sequence similarity.

- Intron conservation information: ExOrthist tries to match each query intron with a target intron in the IPA alignment. Depending on the local protein similarity, the width of the searching window around the query intron changes:

- if $\%$ similarity $\leq 30$ or $\%$ gaps $\geq 30$ : width $=10$

- if $\%$ similarity $\geq 30$ and $\%$ similarity $<50$ : width $=8$

- if $\%$ similarity $\geq 50$ and $\%$ similarity $<70$ : width $=6$

- if $\%$ similarity $\geq 70$ and $\%$ similarity $<80$ : width $=4$

- if $\%$ similarity $\geq 80$ and $\%$ similarity $<90$ : width $=3$

- if $\%$ similarity $\geq 90$ : width $=2$

This window is further increased by the average number of gaps (i.e., "-") within ten positions at each side of the alignment. Then, if a target intron is not found within the search window, conservation of the query intron is set to zero. If a match is found and the phases of the two introns are equal, intron conservation is rated 10. If the phases of the two introns are different, intron conservation is rated - 10 . 
Next, if the two intron positions are not perfectly aligned within the protein sequence alignment, the number of residues by which they are separated in the alignment is subtracted to the intron conservation value. Thus, the maximum intron conservation value is equal to 10 for a perfectly aligned pair of introns in the same phase, and decreases progressively to indicate no intron conservation (0) or presence of non-conserved introns with different phase (negative values). The intron position conservation value is later translated into a partial score used to evaluate pairwise exon orthology relationships. Importantly, intron position conservation is solely evaluated based on the position the introns occupy in the protein alignment and their phases. Other features such as intronic sequence or length are not taken into account, since they are often poorly conserved.

ExOrthist efficiently parallelizes all alignments and realignments thanks to Nextflow's management system, and later joins all the best isoform-wise matches for a given species pair. If the output of a previous ExOrthist run is provided (--prevaln option), the alignment and realignment steps are skipped for all the query and target isoform pairs that have already been aligned.

\section{Scoring and best match selection}

ExOrthist keeps executing the processes separately for each species pair. In here, it selects the best match per gene for each exon in the query species among all the isoform matches of each homologous gene in the target species. Priority is given to the target exons that fulfill the required homology criteria (see below), which are evaluated by five partial scores reflecting the conservation of the previously assessed features of the exon-intron context (section B):

- (1)-(2): Conservation of the (1) upstream and (2) downstream intron phases: ranging $[-0.25,0.25]$, obtained from multiplying the intron conservation value from B by 0.025 . Positive values indicate phase conservation, while deviations from the maximum score reflect shifts in the intron positions within the IPA.

- (3): Conservation of the exon sequence: ranging [0, 0.2]. 0.2 indicates $100 \%$ sequence similarity.

- (4)-(5): Conservation of the (4) upstream and (5) downstream exon sequences: ranging from $[0,0.15] .0 .15$ indicates $100 \%$ sequence similarity.

These partial scores for internal exons are then summed up to generate a global score ranging [0,1]. In the case of first or last exons, for which scores (2) and (5) or (1) and (4) do not exist, respectively, the global score is divided by 0.6 to make it range $[0,1]$. Importantly, the global score is used to select the best representative target isoform per query exon, but is not directly used to infer homology. For the homology inference, different filtering criteria will be applied to each species pair based on their evolutionary distance and the user-specified conservation cut-offs for each of the individual scores. More specifically, the up/downstream intron position conservation, the exon sequence conservation and the up/downstream exon sequence conservation are sequentially evaluated in the filtering. 
- (1) and/or (2), depending on cut-off (b), are required to be positive in all the targetgene best matches (i.e., the intron positions need to be in the same phase and within the allowed window, even if not necessarily in the exact same location). For first and last exons, only (2) and (1) are evaluated, respectively. If the intron phase(s) are conserved, sequence conservation is evaluated.

- (3) is required to be $\geq$ min_ex_sim"0.2, where min_ex_sim is the minimum sequence similarity specified for species1-species2 evolutionary distance range (cutoff $(\mathrm{c}))$.

- (4) and (5) are required to be $\geq$ min_ex_sim*0.15, where min_ex_sim is the minimum sequence similarity specified for species1-species 2 evolutionary distance range (cut-off (c)). For first and last exons, only (5) and (4) are evaluated, respectively.

- Extra filter: the length ratio between two compared exons (shortest/longest) should not be lower than the minimum exon length ratio specified for species1-species2 evolutionary distance range (cut-off (d)).

In the end, among these pre-filtered matches, the match with the highest global score is selected for each query exon and target gene. If no target exon passes all cut-offs, the one with the highest global score is considered the best hit for the target gene. While the ExOrthist's logic requires a query exon to match a unique target exon, each target exon can potentially be matched by multiple query exons, capturing cases of in-tandem exon duplication in the same gene. Moreover, overlapping variants of the query exons (i.e., alternative forms of the same exon due to alternative splice donor/acceptor sites) might be present in the pool of filtered matches. In order to univocally derive orthologous relationships for each exon, ExOrthist selects the form with the highest number of matches (among all target genes) as representative of its overlap group. The best pairwise IPA alignment for a pair of exons can be automatically retrieved using the script retrieve_IPA_aln.pl.

In addition, ExOrthist accepts custom exon orthopairs (from liftOver or manual curation) to be considered in the final exon orthology inference through the --bonafide pairs option. For this purpose, ExOrthist incorporates a custom script (get_liftovers.pl) to allow users to retrieve liftOver-based exon orthopairs for species pairs with available liftOver files. If provided, these exon orthopairs will be given priority over the ExOrthist-inferred matches (i.e., they will be chosen as representatives of their overlap groups) and will be directly integrated in the generation of the exon orthogroups graphs (next section).

\section{Exon clustering}

After deriving exon homologous relationships between each pair of species, ExOrthist works on the combined orthopair information to infer the exon orthogroups. For each gene orthogroup, ExOrthist builds a directed graph (through the $\mathrm{R}$ igraph package v1.2.6 [32]) with exons as nodes and their pairwise homology relationships represented as edges. In case of a best reciprocal match between homologous exons, two directed edges will be drawn between the correspondent nodes. ExOrthist then applies the $\mathrm{R}$ igraph edge-betweenness algorithm to select the optimal graph topology, with 
communities highly intra-connected and lowly interconnected. Although the directionality of the graph is not considered by the edge-betweenness algorithm, the reciprocity of the matches is represented by the number of edges. The exon communities identified in the optimal topology correspond to the multi-species exon orthogroups returned by the pipeline. For each exon, ExOrthist also computes a Membership Score (MS), which reflects its degree of similarity to all the exons belonging to the same orthogroup (OG). The MS is defined as follows:

$\mathrm{MS}=$ (IN_degree + OUT_degree + N_reciprocals) $/(2 *$ (TOT_exons_in_OG - SPE -

CIES_exons_in_OG) + (TOT_genes_in_OG - SPECIES_genes_in_exOG))

with:

- IN_degree: number of exon matches from the other exons in the orthogroup (i.e., the considered exon is target).

- OUT_degree: number of exon matches to the other exons in the orthogroup (i.e., the considered exon is query).

- N_reciprocal: number of reciprocal matches (i.e., query exon is a match of its target exon and vice versa).

- TOT_exons_in_OG: number of exons in the orthogroup.

- SPECIES_exons_in_OG: number of exons from the same species present in the exon orthogroup.

- TOT_genes_in_OG: total number of genes in the original gene orthogroup.

- SPECIES_genes_in_exOG: number of genes from the same species that contribute with exons to the exon orthogroup.

\section{Genome annotation and sequence files}

In order to run ExOrthist, annotation files (GTF) and genome files (fasta) have to be provided for each of the species of interest. For the analyses presented in this study, we downloaded the GTF and genome fasta files from Ensembl (https://www.ensembl.org/), selecting the following assemblies and versions: human (hg38, v88), mouse (mm10, v88), cow (bosTau9, v99), and zebrafish (danRer11, v99). For fruitfly, we downloaded the GTF and fasta genome files from Ensembl Metazoa (dm6, v26). To comply with the UCSC Brower's format, we modified the first field of all GTF files so that the label "chr" was added to the chromosome number (e.g., "1" = > "chr1").

\section{Calibration of ExOrthist's default conservation cut-offs}

We first selected three pairs of species as representatives of each evolutionary distance range. Human-mouse for short (hg38-mm10; estimated divergence: 90 million years ago [MYA] [64]), human-zebrafish for medium (hg38-danRer11; estimated divergence: 435 MYA [64]), and human-fruitfly for long (hg38-dm6; estimated divergence: 797 MYA [64]). We then used Broccoli v1.0 with default settings [18] to infer each pairwise gene orthogroups. We removed all the gene orthogroups containing more than 20 genes, selecting 16,034 orthogroups for hg38-mm10, 11,571 for hg38-danRer11 and 5142 for hg38-dm6.

In order to identify the optimal minimum exon sequence similarity (cut-off or parameter (c)) and minimum exon length ratio (shortest/longest) (cut-off or parameter (d)), 
we systematically analyzed the ExOrthist main (v0.1.0) performance with different combinations of those parameters. First, we kept the minimum length ratio fixed at 0.4 and we performed nine ExOrthist runs for each species pair starting from a minimum sequence similarity of 0.1 and increasing it by 0.1 in each of the following runs up to 0.9 . We then selected the optimal sequence similarity cut-off for the short and medium evolutionary distance ranges depending on when saturation in the number of conserved exons was reached ( 0.5 and 0.3 , respectively) (Fig. $2 \mathrm{~A}-\mathrm{C}$ ). For long evolutionary distances, we selected 0.1 as default sequence similarity cut-off, since saturation was not reached.

Second, we kept the minimum sequence similarity fixed at the identified default parameter for each evolutionary distance range, and we performed five ExOrthist runs for each species pair starting from a minimum exon length ratio of 0.4 and increasing it by 0.1 in each of the following runs up to 0.9. The saturation in the number of conserved exons was reached at an exon length ratio cut-off of 0.6 for both short and medium evolutionary distance ranges. For long evolutionary distances, we selected 0.4 as the default exon length ratio cut-off, since saturation was not reached (Fig. 2D-F). All the mentioned runs were executed with minimum protein similarity $=0.25$ (short), 0.20 (medium), 0.15 (long), and fixed minimum number of conserved introns $=2$

\section{Evaluation of ExOrthist computational performance}

To assess ExOrthist computational performance, we ran the main module (v1.0.0) for the species pairs representative of each evolutionary distance [short: human-mouse (hg38-mm10), medium: human-zebrafish (hg38-danRer11), long: human-fruitfly (hg38dm6)], setting the identified optimal conservation cut-offs (minimum sequence similarity and minimum length ratio; see previous paragraph), fixed number of introns $=2$ and protein similarity $=0.25$ (short), 0.20 (medium), and 0.15 (long). Moreover, to evaluate how the required computational hours and running time scale when launching ExOrthist on more than two species, we performed two extra runs considering three (human: hg38, mouse: mm10, zebrafish: danRer11) and four (human: hg38, mouse: mm10, zebrafish: danRer11, fruitfly: dm6) species at a time. We first used Broccoli v1.0 with default settings [18] to infer gene orthogroups in each set of species. We removed all the gene orthogroups containing more than 20 genes, selecting 15,712 orthogroups for hg38-mm10-danRer11 and 12,509 orthogroups for hg38-mm10-danRer11-dm6. We then ran ExOrthist main (v1.0.0) with the conservation parameters specified above, and by setting the following species pairwise evolutionary distances: short (hg38-mm10), medium (hg38-danRer11, mm10-danRer11), long (hg38-dm6, mm10-dm6, danRer11$\mathrm{dm6}$ ). Lastly, we repeated the runs by providing precomputed pairwise protein alignments through the -prevaln option. Comparisons of the computational performance of these multi-species runs, together with that of species pairwise runs, are reported in Additional file 1: Figure S1.

\section{Comparison of ExOrthist and liftOver performances}

To benchmark ExOrthist at a short evolutionary distance range, we implemented a liftOver-based approach following previous studies [29, 35]. Briefly, we downloaded the liftOver chain alignment files for each pairwise comparison (among human, mouse and 
cow; hg38, mm10 and bosTau9) and performed the liftover for all annotated exons from each species against the other two. As recommended by the developers, we used the following parameters: - minMatch $=0.10$-multiple - minChainT $=200$ -minChainQ $=200$, which we implemented in the script get_liftovers.pl provided as part of ExOrthist. Then, to make the comparisons between ExOrthist and liftOver fairer, we did not require the liftOver matches to have any canonical dinucleotide, and we considered only lifted coordinates that shared a junction with an annotated exon in the target species and in the same gene orthogroups used to run ExOrthist. From these exon pairs, we generated exon orthogroups by running the final scripts of the ExOrthist main module (v0.1.0): D2_cluster_EXs.R and D3_format_ EX_clusters_output.pl.

To obtain ExOrthist exon orthogroups, we used Broccoli v1.0 [18] with default settings to infer gene orthogroups for human, mouse and cow. We filter out 53 orthogroups containing more than 20 genes, restricting the following analysis to 17,594 orthogroups. We ran ExOrthist main (v0.1.0) with conservation cut-offs set at the default values for short distance species (minimum sequence similarity $=0.5$; minimum exon length ratio $=0.6$, minimum global protein similarity $=0.25$, and minimum number of conserved introns $=2$ ).

To compare the exon orthogroups from both approaches, we performed two analyses. First, we asked, for each species and for each approach, how many annotated exons were found in orthogroups with exons from each of the other two species and plotted the overlapping fraction (Fig. 3C). Since exons may have alternative splice acceptor and/or donor sites, we used bedtools intersect to compute the overlap between the two orthogroup files. Second, for exons with a 1:1 match in another species in both exon orthogroup files, we assessed the concordance of these orthology assignments (i.e., whether an exon in species1 was paired to the same exon in species 2 by both approaches), obtaining virtually $100 \%$ concordance.

\section{Investigation of exon evolution after whole genome duplication}

We obtained the 1:2 and 1:1 gene orthogroups between $X$. tropicalis (v9.0) and $X$. laevis (v9.1) from [36] and the respective genome annotations from Xenbase. Genome annotations were downloaded as GFF3 files and converted to GTF format using gffread [65] and custom scripts. We generated exon orthogroups by running ExOrthist main (v0.1.0) with default conservation cut-offs for short evolutionary distance (minimum global protein similarity $=0.25$, number of required introns = 2 , minimum sequence similarity $=0.5$; minimum length ratio $=0.6$ ), and we subsequently quantified the exon orthogroups (1:2 and 1:1) generated from the 8806 1:2 gene orthogroups. More specifically, 1:2 exon orthogroups contained one exon for $X$. tropicalis and its two orthologs in X. laevis (which derived from a whole genome duplication event). Conversely, in 1:1 exon orthogroups, only one $X$. laevis ortholog was detected. Information about the X. laevis subgenome (L or S) was obtained from [36] and was used to parse the 1:1 gene and exon orthogroups to assess in which subgenome the gene/exon ortholog was not detected. We considered an exon to have 1:1 or 1:2 homologs only based on the exon orthogroups and did not take the best hit information into account. 


\section{Comparison of Nova-dependent exon sets}

We used Broccoli v1.0 [18] with default parameters to infer mouse-fruitfly (mm10dm6) gene orthogroups. We selected all the orthogroups (5207) containing less than 20 genes. We ran ExOrthist main (v0.1.0) with default conservation cut-offs for long distance evolutionary range (minimum global protein similarity $=0.15$, number of required introns $=2$, minimum sequence similarity $=0.1$; minimum length ratio $=0.4$ ), adding non-annotated exons identified by vast-tools [29]. To identify Nova-dependent exons, we run vast-tools v2.5.1 for two datasets characterized by deficiency of Nova orthologs in each species. In particular, we used an experiment with Nova2 depletion in mouse embryonic cortex at the E18.5 stage [43] and another one with downregulation of pasilla (ps) in adult fly whole brains [44]. To obtain inclusion levels (PSIs) for all exons, we employed vast-tools align and combine with default parameters for mm10 and dm6 (VASTDB libraries: vastdb.mm2.23.06.20.tar.gz and vastdb.dme.23.06.20.tar.gz) [29]. All replicates for control and depleted samples were merged into a single sample using vast-tools merge to increase read depth, and vast-tools compare with default parameters (minimum change in inclusion levels [min_dPSI] of 15) was employed to define Nova-dependent exons (827 exons in mouse and 407 in fruitfly, respectively; Fig. 5B). Evolutionary comparison of both sets was done using the ExOrthist compare exon_sets (v1.0.0) module with default options and providing two exon sets. The overlap at the gene orthology level between the sets of Nova-regulated exons in the two species was assessed using a hypergeometric test (phyper in R), considering only exons in genes included in the gene orthogroups. The following arguments were inferred: $q$, number of orthogroups with Nova-regulated exons in both species (41); $m$ : number of orthogroups with Nova-regulated exons in mouse (320); $n$ : number of orthogroups without Nova-regulated exons in mouse (5104); $k$ : number of orthogroups with Novaregulated exons in fruifly (193).

Neural regulation (difference in average PSI of neural and non-neural samples) for each Nova-regulated exon was calculated using data from VastDB for mm10 and dm6 [29], and through the script Get_Tissue_Specific_AS.pl [66]. Analysis of splicing-related features was performed with matt cmpr_exons [51] v1.3.0 and a selection of features was plotted in Additional file 1: Figure S3. All the represented features were directly quantified by matt cmpr_exons with the exception of the average intron length, which we obtained by averaging the length of the upstream and downstream introns returned by matt. Control exon sets for each species (constitutive, cryptic, and alternative exons not regulated by Nova) were obtained by filtering the output of vast-tools compare (run with the --print_sets option) to up to 1000 random exons per category. Finally, enrichment of RNA-binding protein motifs was done using matt rna_maps v1.3.0 with the REGEXP motif option for Nova ([CT]CA $[\mathrm{CT}](.\{0,3\}[\mathrm{CT}]\{0,1\} \mathrm{CA}[\mathrm{CT}] \mid .\{4,23\}[\mathrm{CT}] \mathrm{CA}[\mathrm{CT}])\{2\}$, obtained from $[51])$, Rbfox (TGCATG), and IGF2BP2 ([A|C|G][A|C]A[A|C|T][A|T]CA, built by matt rna maps_cisbp from the CISBP-RNA motif M032 [67]).

\section{Supplementary Information}

The online version contains supplementary material available at https://doi.org/10.1186/s13059-021-02441-9.

Additional file 1. Contains Figures S1-S4.

Additional file 2. Contains Table S1 (Orthogroups output from compare_exon_sets).

Additional file 3: Table S2. (Pairwise comparison output from compare_exon_sets). 
Additional file 4. Review history.

\section{Acknowledgements}

We thank Antonio Torres-Mendez for many suggestions and input during the project and Niccolò Arecco for help with the development of exint_plotter. Animal silhouettes were obtained from http://phylopic.org.

\section{Review history}

The review history is available as additional file 4

\section{Peer review information}

Anahita Bishop was the primary editor of this article and managed its editorial process and peer review in collaboration with the rest of the editorial team.

\section{Authors' contributions}

YM and Ml conceived the software. YM developed the core of the main module, with contributions from FM, MI, DB, and SWR. FM developed the exint plotter module. MI wrote the compare exon sets module with input from FM. LC, FM, and JP made the Nextflow implementation. YM, FM, and MI performed computational analyses. FM and MI wrote the manuscript and made the figures, with input from the other authors. All the authors critically reviewed and approved the final manuscript.

\section{Author's information}

Twitter handle: @mirimiam (Manuel Irimia)

\section{Funding}

The research has been funded by the European Research Council (ERC) under the European Union's Horizon 2020 research and innovation program (ERC-StG-LS2-637591 to MI), the Spanish Ministerio de Ciencia (BFU2017-89201-P to MI, including and FPI PhD fellowship to FM), the "Centro de Excelencia Severo Ochoa 2013-2017" (SEV-2012-0208), EMBO Long Term postdoctoral fellowship (ALTF 1505-2015 to YM), and Marie Skłodowska-Curie actions (MSCA) grant (705938 to YM). We acknowledge the support of the CERCA Programme/Generalitat de Catalunya and of the Spanish Ministry of Economy, Industry and Competitiveness (MEIC) to the EMBL partnership.

\section{Availability of data and materials}

The ExOrthist is an open software publicly available under a MIT license on Github (https://github.com/biocorecrg/ ExOrthist) [34] and Zenodo [68]. For the analyses presented in this study, the GTF and genome fasta files from Ensembl (https://www.ensembl.org/) were downloaded, the following assemblies and versions: human (hg38, v88), mouse (mm10, v88), cow (bosTau9, v99) and zebrafish (danRer11, v99) were selected. For fruitfly, the GTF and fasta genome files from Ensembl Metazoa (dm6, v26) were downloaded.

\section{Declarations}

Ethics approval and consent to participate

No ethical approvals were needed for this study.

\section{Competing interests}

The authors declare that they have no competing interests.

\section{Author details}

${ }^{1}$ Centre for Genomic Regulation, Barcelona Institute of Science and Technology, Dr. Aiguader, 88, 08003 Barcelona, Spain. ${ }^{2}$ Universitat Pompeu Fabra, Barcelona, Spain. ${ }^{3}$ Department of Zoology, Charles University, Vinicna 7, 12844 Prague, Czech Republic. ${ }^{4}$ San Francisco State University, 1600 Holloway Ave, San Francisco, CA 94132, USA. ${ }^{5}$ ICREA, Barcelona, Spain.

Received: 22 February 2021 Accepted: 26 July 2021

Published online: 20 August 2021

\section{References}

1. Sanz L, Calvete JJ. Insights into the evolution of a snake venom multi-gene family from the genomic organization of Echis ocellatus SVMP genes. Toxins. 2016;8(7):216. https://doi.org/10.3390/toxins8070216.

2. Cosby RL, Judd J, Zhang R, Zhong A, Garry N, Pritham EJ, et al. Recurrent evolution of vertebrate transcription factors by transposase capture. Science. 2021;371:eabc6405.

3. Grau-Bove X, Ruiz-Trillo I, Irimia M. Origin of exon skipping-rich transcriptomes in animals driven by evolution of gene architecture. Genome Biol. 2018;19(1):135. https://doi.org/10.1186/s13059-018-1499-9.

4. Reyes A, Anders S, Weatheritt RJ, Gibson TJ, Steinmetz LM, Huber W. Drift and conservation of differential exon usage across tissues in primate species. Proc Natl Acad Sci U S A. 2013;110(38):15377-82. https://doi.org/10.1073/pnas.13072 02110

5. Barbosa-Morais NL, Irimia M, Pan Q, Xiong HY, Gueroussov S, Lee LJ, et al. The evolutionary landscape of alternative splicing in vertebrate species. Science. 2012;338(6114):1587-93. https://doi.org/10.1126/science.1230612.

6. Merkin J, Russell CB, Chen P, Burge CB. Evolutionary dynamics of gene and isoform regulation in Mammalian tissues. Science. 2012;338(6114):1593-9. https://doi.org/10.1126/science.1228186. 
7. Torres-Méndez A, Bonnal S, Marquez Y, Roth J, Iglesias M, Permanyer J, et al. A novel protein domain in an ancestral splicing factor drove the evolution of neural microexons. Nature Ecol Evol. 2019;3(4):691-701. https://doi.org/10.1038/ s41559-019-0813-6.

8. Gracheva EO, Cordero-Morales JF, González-Carcacía JA, Ingolia NT, Manno C, Aranguren Cl, et al. Ganglion-specific splicing of TRPV1 underlies infrared sensation in vampire bats. Nature. 2011;476(7358):88-91. https://doi.org/10.1038/na ture10245.

9. Gueroussov S, Gonatopoulos-Pournatzis T, Irimia M, Raj B, Lin ZY, Gingras AC, et al. An alternative splicing event amplifies evolutionary differences between vertebrates. Science. 2015;349(6250):868-73. https://doi.org/10.1126/ science.aaa8381.

10. Tress ML, Abascal F, Valencia A. Alternative splicing may not be the key to proteome complexity. Trends Biochem Sci. 2017.

11. Blencowe BJ. The relationship between alternative splicing and proteomic complexity. Trends Biochem Sci. 2017;42(6): 407-8. https://doi.org/10.1016/j.tibs.2017.04.001.

12. Marlétaz F, Firbas PN, Maeso I, Tena JJ, Bogdanovic O, Perry M, et al. Amphioxus functional genomics and the origins of vertebrate gene regulation. Nature. 2018;564(7734):64-70. https://doi.org/10.1038/s41586-018-0734-6.

13. Gabaldón T, Koonin EV. Functional and evolutionary implications of gene orthology. Nat Rev Genet. 2013;14(5):360-6. https://doi.org/10.1038/nrg3456.

14. Train C, Glover NM, Gonnet GH, Altenhoff AM, Dessimoz C. Orthologous Matrix (OMA) algorithm 2.0: more robust to asymmetric evolutionary rates and more scalable hierarchical orthologous group inference. Bioinformatics. 2017;33(14): i75-82. https://doi.org/10.1093/bioinformatics/btx229.

15. Li L, Stoeckert CJJ, Roos DS. OrthoMCL: identification of ortholog groups for eukaryotic genomes. Genome Res. 2003; 13(9):2178-89. https://doi.org/10.1101/gr.1224503.

16. Miller JB, Pickett BD, Ridge PG. JustOrthologs: a fast, accurate and user-friendly ortholog identification algorithm. Bioinformatics. 2019;35(4):546-52. https://doi.org/10.1093/bioinformatics/bty669.

17. Emms DM, Kelly S. OrthoFinder: phylogenetic orthology inference for comparative genomics. Genome Biol. 2019;20(1): 238. https://doi.org/10.1186/s13059-019-1832-y.

18. Derelle R, Philippe H, Colbourne JK. Broccoli: combining phylogenetic and network analyses for orthology assignment. Mol Biol Evol. 2020:msaa159.

19. Zea DJ, Laskina S, Baudin A, Richard H, Laine E. Assessing conservation of alternative splicing with evolutionary splicing graphs. bioRxiv. 2020. https://doi.org/10.1101/2020.1111.1114.382820.

20. Chakraborty A, Ay F, Davuluri RV. Exon- and Transcript-level mappings for orthologous gene pairs. Bioinformatics. 2021; btab393.

21. Pavesi G, Zambelli F, Caggese C, Pesole G. Exalign: a new method for comparative analysis of exon-intron gene structures. Nucleic Acids Res. 2008;36(8):e47. https://doi.org/10.1093/nar/gkn153.

22. De Moerlooze L, Spencer-Dene B, Revest JM, Hajihosseini M, Rosewell I, Dickson C. An important role for the IIIb isoform of fibroblast growth factor receptor 2 (FGFR2) in mesenchymal-epithelial signalling during mouse organogenesis. Development. 2000;127(3):483-92. https://doi.org/10.1242/dev.127.3.483.

23. Hatje K, Rahman R, Vidal RO, Simm D, Hammesfahr B, Bansal V, et al. The landscape of human mutually exclusive splicing. Mol Syst Biol. 2017;13(12):959. https://doi.org/10.15252/msb.20177728.

24. Irimia M, Maeso I, Gunning PW, Garcia-Fernandez J, Roy SW. Internal and external paralogy in the evolution of Tropomyosin genes in metazoans. Mol Biol Evol. 2010;27(7):1504-17. https://doi.org/10.1093/molbev/msq018.

25. Hinrichs AS, Karolchik D, Baertsch R, Barber GP, Bejerano G, Clawson H, et al. The UCSC Genome Browser Database: update 2006. Nucleic Acids Res. 2006;34(90001):D590-8. https://doi.org/10.1093/nar/gkj144.

26. Tommaso PD, Chatzou M, Floden EW, Barja PP, Palumbo E, Notredame C. Nextflow enables reproducible computational workflows. Nat Biotechnol. 2017;35(4):316-9. https://doi.org/10.1038/nbt.3820

27. Trapnell C, Roberts A, Goff L, Pertea G, Kim D, Kelley DR, et al. Differential gene and transcript expression analysis of RNA-seq experiments with TopHat and Cufflinks. Nat Protoc. 2012;7(3):562-78. https://doi.org/10.1038/nprot.2012.016.

28. Vaquero-Garcia J, Barrera A, Gazzara MR, Gonzalez-Vallinas J, Lahens NF, Hogenesch JB, et al. A new view of transcriptome complexity and regulation through the lens of local splicing variations. Elife. 2016:5:e11752. https://doi org/10.7554/eLife.11752.

29. Tapial J, Ha KCH, Sterne-Weiler T, Gohr A, Braunschweig U, Hermoso-Pulido A, et al. An atlas of alternative splicing profiles and functional associations reveals new regulatory programs and genes that simultaneously express multiple major isoforms. Genome Res. 2017;27(10):1759-68. https://doi.org/10.1101/gr.220962.117.

30. Shen S, Park JW, Lu ZX, Lin L, Henry MD, Wu YN, et al. rMATS: robust and flexible detection of differential alternative splicing from replicate RNA-Seq data. Proc Natl Acad Sci U S A. 2014;111(51):E5593-601. https://doi.org/10.1073/pna s.1419161111.

31. Sterne-Weiler T, Weatheritt RJ, Best AJ, Ha KCH, Blencowe BJ. Efficient and accurate quantitative profiling of alternative splicing patterns of any complexity on a laptop. Mol Cell. 2018;72(1):187-200. https://doi.org/10.1016/j.molcel.2018.08.018

32. Csardi G, Nepusz T. The igraph software package for complex network research: InterJournal, Complex Systems; 2006.

33. Irimia M, Roy SW. Spliceosomal introns as tools for genomic and evolutionary analysis. Nucleic Acids Res. 2008;36(5): 1703-12. https://doi.org/10.1093/nar/gkn012.

34. Marquez Y, Mantica F, Cozzuto L, Burguera D, Hermoso-Pulido A, Ponomarenko J, et al. ExOrthist: a tool to infer exon orthologies at any evolutionary distance. Github. 2021; https://github.com/biocorecrg/ExOrthist.

35. Irimia M, Weatheritt RJ, Ellis J, Parikshak NN, Gonatopoulos-Pournatzis T, Babor M, et al. A highly conserved program of neuronal microexons is misregulated in autistic brains. Cell. 2014;159(7):1511-23. https://doi.org/10.1016/j.cell.2014.11.035

36. Session AM, Uno Y, Kwon T, Chapman JA, Toyoda A, Takahashi S, et al. Genome evolution in the allotetraploid frog Xenopus laevis. Nature. 2016;538(7625):336-43. https://doi.org/10.1038/nature19840.

37. Kalsotra A, Cooper TA. Functional consequences of developmentally regulated alternative splicing. Nat Rev Genet. 2011; 12(10):715-29. https://doi.org/10.1038/nrg3052.

38. Li Q, Lee JA, Black DL. Neuronal regulation of alternative pre-mRNA splicing. Nat Rev Neurosci. 2007:8(11):819-31. https://doi.org/10.1038/nrn2237. 
39. Sebestyen E, Singh B, Minana B, Pages A, Mateo F, Pujana MA, et al. Large-scale analysis of genome and transcriptome alterations in multiple tumors unveils novel cancer-relevant splicing networks. Genome Res. 2016; Epub ahead of print.

40. Parikshak NN, Swarup V, Belgard TG, Irimia M, Ramaswami G, Gandal MJ, et al. Genome-wide changes in IncRNA, splicing, and regional gene expression patterns in autism. Nature. 2016;540(7633):423-7. https://doi.org/10.1038/nature2 0612.

41. Elorza A, Marquez Y, Cabrera JR, Sanchez-Trincado JL, Santos-Galindo M, Hernandez IH, et al. Huntington's diseasespecific mis-splicing unveils key effector genes and altered splicing factors. Brain. 2021;awab087.

42. Irimia M, Rukov JL, Roy SW, Vinther J, Garcia-Fernandez J. Quantitative regulation of alternative splicing in evolution and development. Bioessays. 2009;31(1):40-50. https://doi.org/10.1002/bies.080092.

43. Saito Y, Miranda-Rottmann S, Ruggiu M, Park CY, Fak JJ, Zhong R, et al. NOVA2-mediated RNA regulation is required for axonal pathfinding during development. eLife. 2016;5:e14371. https://doi.org/10.7554/eLife.14371.

44. Sapiro AL, Freund EC, Restrepo L, Qiao H, Bhate A, Li Q, et al. Zinc finger RNA-binding protein Zn72D regulates ADARmediated RNA editing in neurons. Cell Rep. 2020;31(7):107654. https://doi.org/10.1016/j.celrep.2020.107654.

45. Brooks AN, Yang L, Duff MO, Hansen KD, Park JW, Dudoit S, et al. Conservation of an RNA regulatory map between Drosophila and mammals. Genome Res. 2011;21(2):193-202. https://doi.org/10.1101/gr.108662.110.

46. Irimia M, Denuc A, Burguera D, Somorjai I, Martín-Durán JM, Genikhovich G, et al. Stepwise assembly of the novaregulated alternative splicing network in the vertebrate brain. Proc Natl Acad Sci U S A. 2011;108(13):5319-24. https:// doi.org/10.1073/pnas.1012333108.

47. Solana J, Irimia M, Ayoub S, Orejuela MR, Zywitza V, Jens M, et al. Conserved functional antagonism of CELF and MBNL proteins controls stem cell-specific alternative splicing in planarians. eLife. 2016;5:e16797. https://doi.org/10.7554/eLife.1 6797.

48. Burguera D, Marquez Y, Racioppi C, Permanyer J, Torres-Mendez A, Esposito R, et al. Evolutionary recruitment of flexible Esrp-dependent splicing programs into diverse embryonic morphogenetic processes. Nat Commun. 2017;8(1):1799. https://doi.org/10.1038/s41467-017-01961-y.

49. Ule J, Ule A, Spencer J, Williams A, Hu JS, Cline M, et al. Nova regulates brain-specific splicing to shape the synapse. Nat Genet. 2005;37(8):844-52. https://doi.org/10.1038/ng1610.

50. Seshaiah P, Miller B, Myat MM, Andrew DJ. pasilla, the Drosophila homologue of the human Nova-1 and Nova-2 proteins, is required for normal secretion in the salivary gland. Dev Biol. 2001;239(2):309-22. https://doi.org/10.1006/ dbio.2001.0429.

51. Gohr A, Irimia M. Matt: Unix tools for alternative splicing analysis. Bioinformatics. 2019;35(1):130-2. https://doi.org/10.1 093/bioinformatics/bty606.

52. Ule J, Stefani G, Mele A, Ruggiu M, Wang X, Taneri B, et al. An RNA map predicting Nova-dependent splicing regulation. Nature. 2006;444(7119):580-6. https://doi.org/10.1038/nature05304.

53. Zhang C, Frias MA, Mele A, Ruggiu M, Eom T, Marney CB, et al. Integrative modeling defines the nova splicing-regulatory network and its combinatorial controls. Science. 2010;329(5990):439-43. https://doi.org/10.1126/science.1191150.

54. Roy SW, Fedorov A, Gilbert W. Large-scale comparison of intron positions in mammalian genes shows intron loss but no gain. Proc Natl Acad Sci U S A. 2003;100(12):7158-62. https://doi.org/10.1073/pnas.1232297100.

55. Roy SW, Hartl DL. Very little intron loss/gain in Plasmodium: intron loss/gain mutation rates and intron number. Genome Res. 2006;16(6):750-6. https://doi.org/10.1101/gr.4845406.

56. Csuros M, Rogozin IB, Koonin EV. A detailed history of intron-rich eukaryotic ancestors inferred from a global survey of 100 complete genomes. PLoS Comput Biol. 2011;7(9):e1002150. https://doi.org/10.1371/journal.pcbi.1002150.

57. Coulombe-Huntington J, Majewski J. Intron loss and gain in Drosophila. Mol Biol Evol. 2007;24(12):2842-50. https://doi. org/10.1093/molbev/msm235.

58. Denoeud F, Henriet S, Mungpakdee S, Aury JM, Da Silva C, Brinkmann H, et al. Plasticity of animal genome architecture unmasked by rapid evolution of a pelagic tunicate. Science. 2010;330(6009):1381-5. https://doi.org/10.1126/science.11 94167.

59. Huff JT, Zilberman D, Roy SW. Mechanism for DNA transposons to generate introns on genomic scales. Nature. 2016; 538(7626):533-6. https://doi.org/10.1038/nature20110.

60. Roy SW, Gilbert W. Complex early genes. Proc Natl Acad Sci U S A. 2005;102(6):1986-91. https://doi.org/10.1073/pnas.04 08355101.

61. Gelfman S, Burstein D, Penn O, Savchenko A, Amit M, Schwartz S, et al. Changes in exon-intron structure during vertebrate evolution affect the splicing pattern of exons. Genome Res. 2012;22(1):35-50. https://doi.org/10.1101/gr.119834.110.

62. Alekseyenko AV, Kim N, Lee CJ. Global analysis of exon creation versus loss and the role of alternative splicing in 17 vertebrate genomes. RNA. 2007;13(5):661-70. https://doi.org/10.1261/rna.325107.

63. Katoh K, Standley DM. MAFFT multiple sequence alignment software version 7: improvements in performance and usability. Mol Biol Evol. 2013;30(4):772-80. https://doi.org/10.1093/molbev/mst010.

64. Kumar S, Stecher G, Suleski M, Hedges SB. TimeTree: a resource for timelines, timetrees, and divergence time. Mol Bio Evol. 2017;34(7):1812-9. https://doi.org/10.1093/molbev/msx116.

65. Pertea G, Pertea M. GFF Utilities: GffRead and GffCompare. F1000Res. 2020;9:304.

66. Martín G, Márquez Y, Mantica F, Duque P, Irimia M. Alternative splicing landscapes in Arabidopsis thaliana across tissues and stress conditions highlight major functional differences with animals. Genome Biol. 2021;22(1):35. https://doi.org/1 0.1186/s13059-020-02258-y.

67. Ray D, Kazan H, Cook KB, Weirauch MT, Najafabadi HS, Li X, et al. A compendium of RNA-binding motifs for decoding gene regulation. Nature. 2013;499(7457):172-7. https://doi.org/10.1038/nature12311.

68. Marquez Y, Mantica F, Cozzuto L, Burguera D, Hermoso-Pulido A, Ponomarenko J, et al. ExOrthist: a tool to infer exon orthologies at any evolutionary distance. Zenodo. 2021.

\section{Publisher's Note}

Springer Nature remains neutral with regard to jurisdictional claims in published maps and institutional affiliations. 